\title{
Scanning the welded seams of titanium alloys by using subminiature eddy current transducers
}

\author{
Sergey Dmitriev ${ }^{1}$, Vladimir Malikov ${ }^{1, *}$, and Anatoly Sagalakov ${ }^{1}$ \\ ${ }^{1}$ Altai State University, Faculty physics and technology, 65604 Barnaul, Russia
}

\begin{abstract}
The eddy current transducer (ECT) of the transformer type is used to construct a sensor for investigating titanium sheets joined by a welded joint. The characteristics of the ECT are presented. The measurement method for controlling the occurrence of defects in the welded joints in titanium alloys is described. The experimental results obtained by the ECT on two welded titanium sheets are presented. The depth of penetration of the field of eddy currents into the investigated object is determined and the dependences describing the response of the ECT at different depths of the defect are outlined. The relationships can be used for evaluating the quality of welded joints and determining the reliability of welding.
\end{abstract}

\section{Introduction}

It is well-known that many inspection methods describe the violation of the suitability of an object for further application. Therefore, they can be used only for checking individual items in a production batch [1]. Alternative methods of non-destructive eddy current inspection can be used for testing all components so that the quality can be determined accurately.

The problem of evaluating the quality of welding and welded joints in different metals has been discussed many times in different scientific journals. For example, in [2], experiments were carried out to evaluate the relative strength of a welded joint and a parent metal. In [3], the authors propose a new method of evaluating the parameters of the quality of welded joints based on the effect of a high-speed waterjet on the surface of the inspected object.

An urgent problem of non-destructive inspection in engineering is the determination of the quality of welded joints in titanium alloys. Titanium and its joints are used in large volumes in aircraft construction, shipbuilding, chemical engineering and important machine components. Commercial titanium is used for the manufacture of components designed for operation in corrosive media. However, low-quality welded joints in titanium may result in the fracture of components produced from this material. The application of eddy current flaw detection for evaluating the quality of welded joints in titanium alloys is an important task requiring urgent solution. Since the eddy current inspection method is not sensitive to non-conducting paint layers, it can be used for the diagnostics of components with lacquer and varnish coatings. The currently available eddy current transducers (ECTs) can be used to determine the quality of welded joints with a high degree of accuracy [4]. The experiments carried out in this work show that the method can be used for evaluating the homogeneity of material of a welded joint.

One of the well-known shortcomings of eddy current flaw inspection is the inspection of the quality of thin surface layers of electrically conducting materials. The design of the ECT, the small dimensions and the special form of the core allow defects to be found at depths of up to $5 \mathrm{~mm}$.

\section{Description of the system}

We have developed a superminiature eddy-current transformer for local monitoring of the physical parameters in titanium-alloy plates and weld seams [5]. In contrast to existing sensors, it permits local measurements with sections measuring a few microns, to depths of the order of $5 \mathrm{~mm}$. The electrical conductivity of the alloy is directly measurable, and its distribution over the sample surface and thickness may be readily established. The eddy-current method is based on the dependence of the current magnitude and distribution on the geometric and electromagnetic parameters of the sample and on the mutual position of the sensor and the sample. The basic informative parameter $\beta_{0}$ is a generalized characteristic of the object, the eddy-current sensor, and the frequency of the electromagnetic field [5]. We need to develop an adequate model of the response of a eddy-current transformers of plate type, which are sensitive to many variables and permit reproduction of the voltage hodograph at small values of $\beta_{0}$. We have plotted hodographs illustrating the influence of various parameters on the induced voltage on the basis of the proposed model [5]. The eddy-current transformer is connected to the sound board of a

\footnotetext{
* Corresponding author: osys11@gmail.com
} 
computer with special software that controls the voltage at the transformers exciting winding and also reads the voltage at the measuring winding (in arbitrary units). On the basis of preliminary calibration, these readings are converted to values of the electrical conductivity. The exciting winding (diameter $\mathrm{D}_{1}=0.12-0.13 \mathrm{~mm}$ ) of the superminiature eddy-current transformer consists of ten turns of copper wire (cross-sectional area $5 \mu \mathrm{m}^{2}$ ). The measuring winding (diameter $0.05-0.08 \mathrm{~mm}$ ) consists of 130 turns of copper wire (cross-sectional area $20 \mu \mathrm{m}^{2}$ ). To minimize the influence of the exciting winding on the final signal, the circuit includes a compensation winding that consists of 20 turns of copper wire (crosssectional area $5 \mu \mathrm{m}^{2}$ ), connected to the measuring winding in such a way that the voltage of the exciting winding is subtracted from the result. The windings are wrapped around a core of $2000 \mathrm{NMZ}$ ferrite (relative magnetic permeability $\mu_{\max }=500$ or else of 81NMA alloy annealed by a special method (if greater localization of the magnetic field is required). The core consists of a tetrahedral pyramid (height $1 \mathrm{~mm}$ ), with a square base (sides of $0.2 \mathrm{~mm}$ ). The measuring winding rests on the points of the pyramid, which improves the localization of the magnetic field.

The eddy-current transducer (Figure 1) is a transformer with measuring (1), exciting (2), and compensation (3) windings and a magnetic circuit 4, which is located inside the cylindrical platform 5 with tracks that are cut on the external side for windings. The platform is impregnated with a compound 6 at a temperature of $200^{\circ} \mathrm{C}$ to prevent the disintegration of the windings when the ferrite screen 7 , which is intended for the localization of the electromagnetic field on the tested object, is put in place. From the outside the transducer is contained in a corundum washer 8 , which protects the core 4 from contacting the tested object.

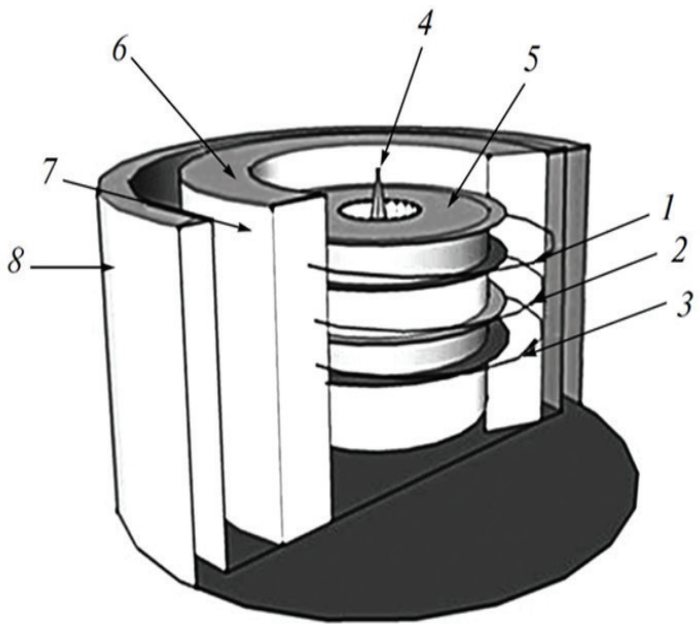

Fig. 1. Scheme of eddy current transducer

Such transformers permit effective localization of the magnetic field, so that defects as small as $250 \mu \mathrm{m}$ may be detected. In addition, the magnetic field penetrates into the sample to a considerable depth when working at relatively low frequencies. The corresponding software is written in the language $\mathrm{C}++$ for the Windows operating system. By means of the Windows mixer subsystem, the software controls the voltage applied to the exciting winding, thereby specifying the amplitude and frequency of the sinusoidal digital signal from the virtual generator.

\section{Operation of the measuring system}

The digital signal from the virtual generator is sent to the input of the digital-analog converter of the sound card. The analog signal then passes through a power amplifier to the exciting winding of the eddy current transformer. The sinusoidal signal creates an electromagnetic field, which, on interacting with the sample, induces an emf in the measuring winding of the eddy-current transformer. This voltage is sent through a preamp to the microphone input of the sound card and then to the input of the analog-digital converter in the sound card. The resulting digital signal is sent to the analysis and control module of the software. This module determines the magnitude of the digital signal (in conventional units) corresponding to the voltage $\mathrm{U}_{\mathrm{m}}$ at the measuring winding.

The computer's sound card permits variation in frequency of the electromagnetic field created by the exciting winding within the range $100-2000 \mathrm{~Hz}$ in the course of scanning.

\section{Results and discussion}

A set of measurements was performed on VT1-0 technical titanium plates that were connected by welded seams to demonstrate the appropriateness of the proposed unit for the determination of the quality of welded seams. The plate thickness was $5 \mathrm{~mm}$ and the width of the welded seam was $5 \mathrm{~mm}$. Before the measurements were started, the transducer was calibrated. The calibration consisted of the determination of the induced voltage from the flawfree sector. The measured characteristic was the voltage that is induced by the field of eddy currents that arise in the tested object. In this experiment a sector for calibration was selected on an intentionally flawfree plate that was manufactured from an identical titanium plate. The calibration was performed at different frequencies. The frequency was varied from 500 to $2000 \mathrm{~Hz}$ with a $100 \mathrm{~Hz}$ step. The subsequent scanning was carried out by displacing the transducer along or across the welded seam or across the flaw area. During the experiments it was determined that the optimal frequency range of the electromagnetic field of the exciting winding for studying titanium is approximately $1500 \mathrm{~Hz}$.

The scanning lengthwise of the weld seam surface was conducted in order to determine the weld seam homogeneity. Herewith, it should be noted that any significant change in the signal amplitude wasn't pointed out. The results of the experiment are presented in Fig. 2. 


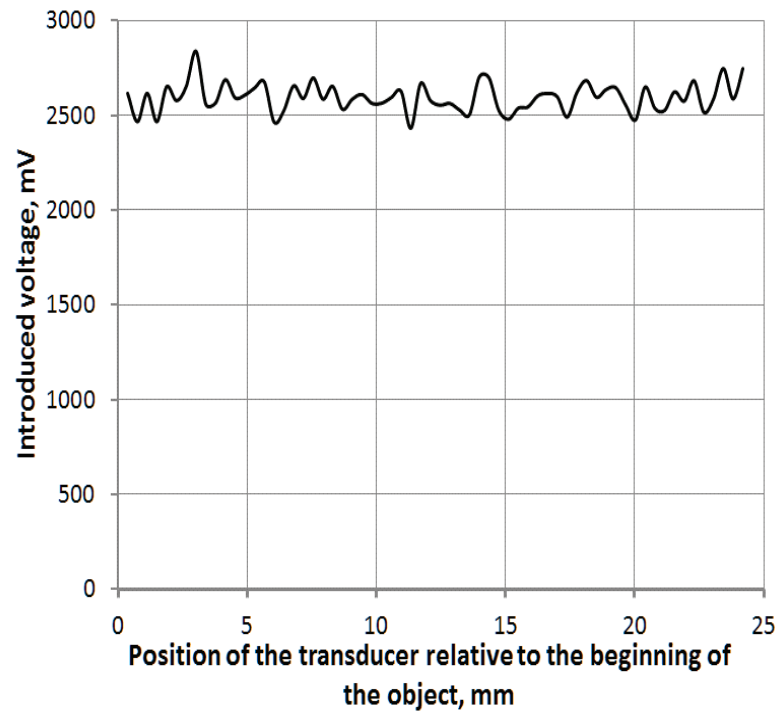

Fig.2. The response value during the scanning lengthwise of the welded seam.

The results of the experiment say for the relatively homogeneous structure of the weld seam but don't provide us with any information regarding the quality of the seam itself. Judging by the results of the given experiment, it could be concluded about the equal distribution of the weld seam defects or its zero-defect quality only.

The scanning was conducted crosswise of the weld seam in the next experiment. The length of the weld seam was $150 \mathrm{~mm}$. The seam was divided into 30 sections, $5 \mathrm{~mm}$ each, so that the signal from the seam itself as well as the one coming directly from the slabs was withdrawn. The dependences received were averaged. The results of the experiment are presented in Fig. 3,4.

In the specimen given, the influence of a deficient weld seam on the inserted voltage is clearly traced by a significant drop in the signal amplitude in the weld seam area in comparison with the slabs area.

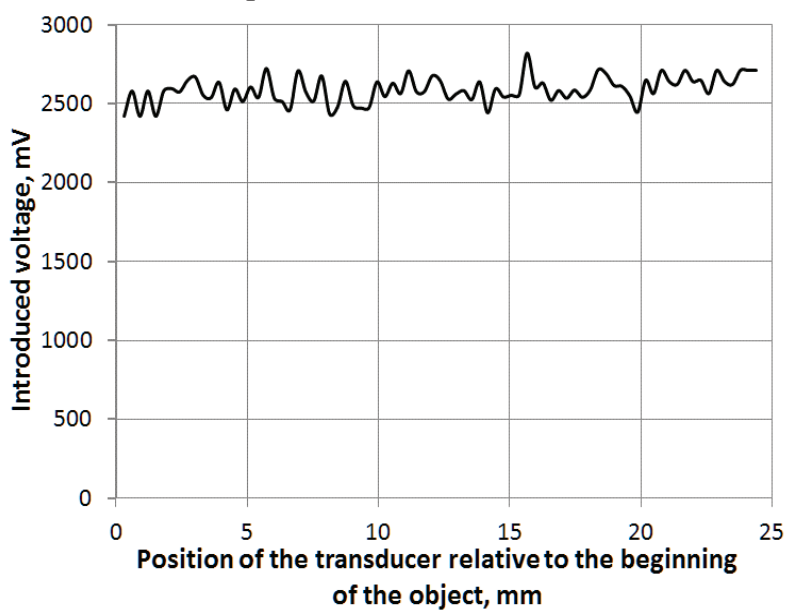

Fig. 3. The response value during the scanning lengthwise of the welded seam Specimen №1

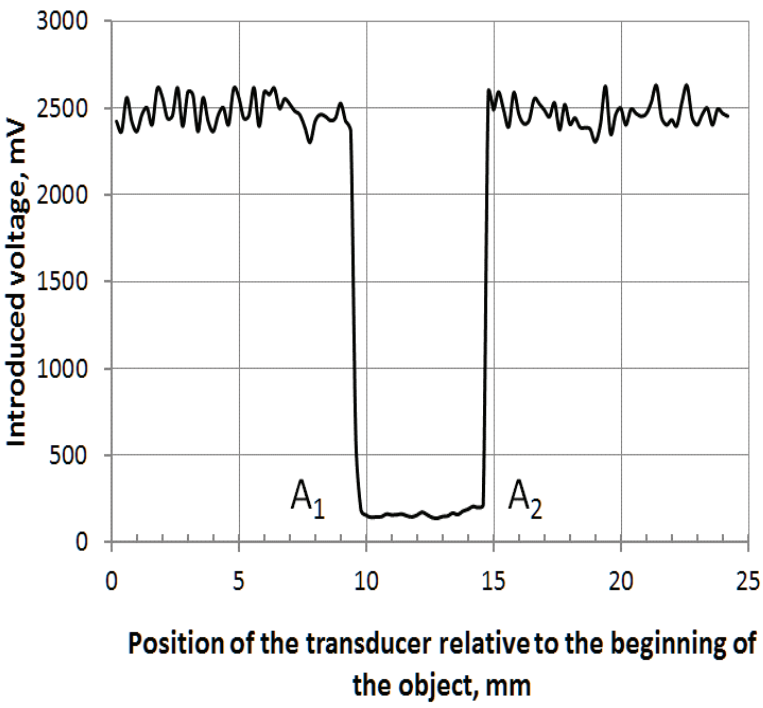

Fig. 4. The response value during the scanning of the welded seam: (a) sample no. 1, A1-A2 are the boundaries of the welded seam, and (b) sample no. 2.

The conclusion about the weld seam of Specimen 1 low quality was made based on the experiment conducted. The low quality of the welding was confirmed directly by the weld seam cut as well. Specimen 2 scanning showed the absence of the signal amplitude excursion within the frame of the weld seam. The weld seam of Specimen 2 cut showed the high quality of welding.

Additional experiments were carried out in simulation of a similar amplitude drop. In these experiments, two titanium sheets of the same thickness were placed in tight contact and subsequently the interface was scanned with a scanning frequency of 1600 Hz. The results of the experiment are presented in Fig. 5. These experiments yielded dependence identical to that shown in Fig. 4 in the region of the welded joint in the specimen. The signal amplitude in the region of the interface decreased by more than an order of magnitude in comparison with the signal amplitude from the sheets.

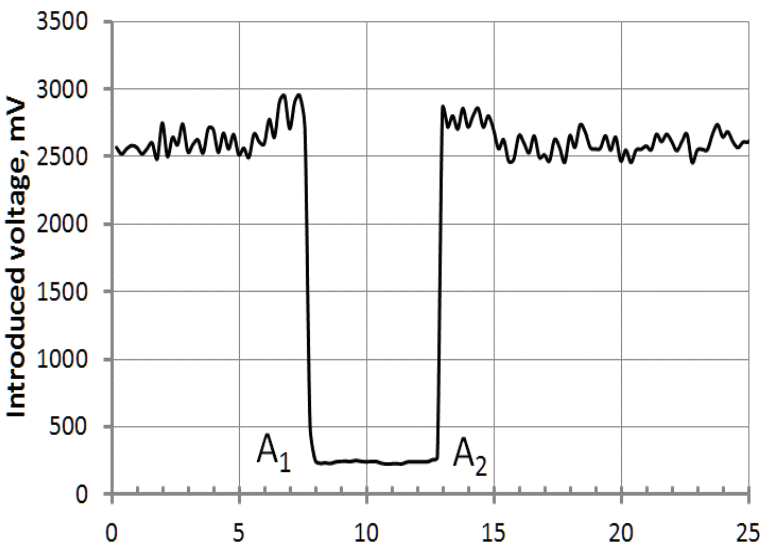

Position of the transducer relative to the beginning of the object, $\mathrm{mm}$

Fig. 5. The response value during the scanning in the area of two slabs junction. A1 - A2 correspond to the edges of the area where the junction presence influences the inserted voltage. 


\section{Conclusions}

The results of the experiments conducted showed a high efficiency of the developed method in the search for defects of the titanium slabs weld seams and in the evaluation of the welding quality. Thanks to the change of the eddy-current transducer signal amplitude in the area of the weld seam, we succeeded in defining the edges of the weld seam exactly, its low quality being obvious due to a steep drop in the signal amplitude. Likewise, the dependence, received as a result of Specimen 2, characterized by a high welding quality, scanning, showed the absence of any significant signal amplitude changes.

So, the analysis of the eddy-current transducer response can be used for the evaluation of the performed welding quality.

\section{References}

1. S. Dmitriev, A Sagalakov, Polz. Vest. 2, 45 (2010)

2. V. Bulychev, R. Latypov, Svar. Proiz. 6, 120 (2012)

3. M. Abashin, Svar. Proiz. 9, 57 (2014)

4. V. Polyakov, V. Malikov, A. Ishlov, S. Dmitriev, Adv Mater Res. 880, 105 (2014)

5. S. Dmitriev, L. Dmitrieva, V. Malikov, A. Sagalakov, IOP Conf. S.: Mat. Sci. Eng. 116, 1 (2015) 